\title{
ERYTHRODERMA - A CLINICAL STUDY OF 43 CASES
}

\author{
Sangameshwara G. M11, Lohith Shivappa², Ganga Huvin ${ }^{3}$
}

${ }^{1}$ Associate Professor, Department of Dermatology, Subbaiah Institute of Medical Sciences.

${ }^{2}$ Assistant Professor, Department of ENT, Subbaiah Institute of Medical Sciences.

${ }^{3}$ Assistant Professor, Department of Pathology, Subbaiah Institute of Medical Sciences.

ABSTRACT

\section{BACKGROUND}

Erythroderma is a cutaneous reaction pattern characterised by generalised erythema and scaling which can be seen in wide range of cutaneous or systemic diseases.

Aim - The study is undertaken to evaluate the clinical profile, aetiology of erythroderma and to correlate the clinical diagnosis with histopathological findings.

\section{MATERIALS AND METHODS}

Forty-three cases of erythroderma were taken for the study from June 2015 to December 2016. These patients were attending outpatient and inpatient department at Subbaiah Medical College, Shimoga.

\section{RESULTS}

Erythroderma seen in forty-three cases. Incidence in male was $75 \%$ and in female $25 \%$. Pre-existing dermatoses was common $(79.07 \%)$ followed by drug reaction (11.6\%), idiopathic cause (6.97\%), malignancy (2.34\%). Common symptoms were fever, malaise observed (40\%), moderate itching, generalised erythema, scaling, burning sensation present in all cases, oedema of the limbs (14\%), lymphadenopathy (33\%). Laboratory investigation shows anaemia (32.5\%), leucocytosis (39.53\%), ESR more than $15 \mathrm{~mm} / \mathrm{hr}$. (44.2\%). Histopathology showed specific changes (13.9\%).

\section{CONCLUSION}

Aetiological factor of erythroderma may show geographical variations. Erythroderma secondary to pre-existing dermatoses was common. Idiopathic, induced erythroderma showed low percentage. The clinical features of erythroderma were identical, irrespective of aetiology. The onset of disease was insidious except in drug-induced erythroderma which was acute. The group that associated with best prognosis was the one related to drug.

\section{KEYWORDS}

Erythroderma, Drug Reaction, Pre-existing Dermatoses.

HOW TO CITE THIS ARTICLE: Sangameshwara GM, Shivappa L, Huvin G. Erythroderma - A clinical study of 43 cases. J. Evolution Med. Dent. Sci. 2017;6(6):461-464, DOI: 10.14260/Jemds/2017/101

\section{BACKGROUND \\ Erythroderma is a rare skin disorder characterised by} erythema and scaling involving $90 \%$ and more of the body surface area. Erythroderma is not a specific diagnosis but clinical manifestation of variety of underlying diseases. It is more common in males and is usually seen in elderly patients; however, age of onset is primarily related to aetiology. Most cases of erythroderma in young patients are due to atopic dermatitis. Many times, erythroderma is the manifestation of an underlying skin disorder like psoriasis, atopic dermatitis, pityriasis rubra pilaris or actinic dermatosis. It may be related to drug intake, idiopathic or due to malignancies. It usually evolves slowly over months to years except for drug reactions and atopy, which tends to develop more acutely.

Financial or Other, Competing Interest: None.

Submission 03-12-2016, Peer Review 03-01-2017,

Acceptance 11-01-2017, Published 19-01-2017.

Corresponding Author:

Dr. Sangameshwara G. M,

Associate Professor,

Department of Dermatology,

Subbaiah Institute of Medical Sciences,

NH 17, Purle-577222,

Shivamogga, Karnataka.

E-mail: sangameswara.1611@gmail.com

DOI: $10.14260 /$ jemds $/ 2017 / 101$
Common symptoms include fever, chills, malaise and pruritus along with peripheral oedema, lymphadenopathy and secondary skin infections. It is important to establish correct diagnosis because specific therapy other than corticosteroids or anti-inflammatory treatment may be necessary to improve patient's condition. Erythroderma carries a significant risk of mortality and morbidity and accounts for up to $1 \%$ of all dermatological hospital admissions. 1

Many of the authors are of the opinion that the causative factors can be grouped as 1) Pre-existing dermatoses 2) Drug reactions 3) Malignancies 4) Systemic disease 5) infection 6) Idiopathic disorders. ${ }^{2}$

Shegal and Srivastava recoded the incidence of erythroderma in a large prospective study from Indian subcontinent as 35 per 100,000 dermatologic outpatients. ${ }^{2}$

The four more common causes of idiopathic protracted erythroderma are probably atopic dermatitis of the elderly, intake of drugs overlooked by the patients, prelymphomatous eruptions and occult malignancies. ${ }^{2}$ Histopathology can help to identify the cause of erythroderma in up to $50 \%$ of cases particularly skin biopsies. ${ }^{2}$ Many chronic dermatoses may be histopathologically indistinguishable in erythrodermic patients. ${ }^{3}$ 
In Pakistan, Pal and Haroon have studied the features of erythroderma in 90 patients and aetiologically found preexisting dermatoses are the common cause of erythroderma. ${ }^{4}$

Common symptoms include fever, chills, malaise, erythema with scaling, and pruritus along with peripheral oedema, lymphadenopathy and secondary skin infection. Visceral involvement may be seen. It is important to establish correct diagnosis because of specific therapy other than corticosteroids or anti-inflammatory treatment may be necessary to improve patient's condition. Erythroderma carries a risk of mortality and morbidity and accounts for up to $1 \%$ of all dermatological hospital admissions. ${ }^{2}$

\section{MATERIALS AND METHODS}

Ethical committee approval from the institution was obtained and informed consent from the patient was taken. Outpatient and inpatients with well-established and clinically typical cases where chosen. All forty-three cases of erythroderma patients had $90 \%$ of body surface involvement with clinically well established, typical cases taken for the present study. A detailed history was taken about onset and evolution of the disease and common symptoms were recorded. Cutaneous and systemic examination was done.

Findings were recorded in in the proforma for analysis and interpretation of data. Data collected was compared with similar studies conducted elsewhere.

\section{RESULTS}

Forty-three cases of erythroderma were noticed out of which thirty-two were male (75\%), eleven cases were female (25\%). Out of forty-three cases of erythroderma, one patient was in the age group of 11-20 years, three patients were in the age group 21 to 30 years, six patients were in the age between 31 to 40 years, nineteen patients were in the age group between 41 to 50 years, ten patients were in the age group between 51 to 60 years. Four patients were above the age of 61 years.

Pre-existing dermatoses constitute thirty-four cases (79.07\%). Psoriasis was seen in sixteen cases, airborne allergic contact dermatitis was noticed in nine cases, two cases each noticed in lichen planus and scabies, one case each was seen in ichthyosis, phototoxic dermatitis, pemphigus foliaceus, pityriasis rubra pilaris, dermatophytosis. Druginduced erythroderma was observed in five cases (11.6\%) two were carbamazepine-induced cases, one each seen in Analgin, cotrimoxazole, Dapsone-induced cases. Idiopathic cause was noticed in three cases (6.97\%), malignancy was noted in one case of Hodgkin's lymphoma (2.34\%) (Table-1). Past history of skin disease is seen in 29 cases. Drug-induced and idiopathic cases had no past history of skin disease.

Common symptoms like fever, malaise, seen in seventeen cases. Other common findings were moderate itching, generalised erythema, scaling, burning sensation seen in all cases. Six patients had oedema of limb, lymphadenopathy seen in fourteen cases. Hepatomegaly with jaundice and oral mucosa involvement was seen in one case and was due to Dapsone-induced reaction. Past history of dermatoses noted in twenty-nine cases.

Anaemia was noticed in fourteen cases- normocytic normochromic anaemia was seen in six cases, macrocytic anaemia was seen in five cases, dimorphic anaemia was seen in three cases. Leucocytosis more than 11000 cells/cu. mm was noticed in seventeen cases. In all these cases, neutrophils and eosinophils were more than normal. ESR more than 15 $\mathrm{mm} / \mathrm{hr}$. was noticed in nineteen cases. Histopathology - skin biopsy showed nonspecific change in thirty-seven cases, specific change was seen in six cases- three in psoriasis, two in lichen planus and one in pemphigus foliaceus respectively (Table- 2).

\section{DISCUSSION}

The approach to patients with erythroderma depends on their dermatological history. Patient's dermatologic disorders recalcitrant to therapy may develop erythroderma during a flare-up. In such cases, the aetiological diagnosis is easy to establish, otherwise, erythroderma remains a diagnostic challenge. The clinical feature of erythroderma is nonspecific and certain clues such as scaling or pruritus could not be related to any specific cause. Erythroderma of long duration may cause hair loss or nail dystrophy regardless of origin, so these changes are also nonspecific. In erythrodermic patients clinicopathological correlation is usually poor, because the specific cutaneous changes of dermatoses or drug reaction are obscured by the nonspecific changes induced by the inflammatory process of erythroderma..$^{2}$ In a patient without history of dermatologic diseases and who denies having recently taken any medication, the diagnosis is more difficult and it is of great importance to perform skin biopsies in such cases although histologic picture shows either a subacute or chronic dermatitis and psoriasiform reaction. Thus, each case of undermined origin requires thorough histological examination, thorough multiple skin biopsies and a lymph node biopsy to rule out lymphoma. 5 In our study, the incidence was more in males i.e. thirty-two cases (75\%), in females it was seen in eleven cases (25\%). In this study, one patient had erythroderma in the age group of 11-20 years, three patients were in the age group of 21-30 years, six patients were in the age group of 31-40 years, nineteen patients were in the age group of 41-50 years, ten patients were in the age group of 51-60 years, and in above 61 years four patients were noted. 29 cases of erythroderma were in the age group of 41-60 years while 10 cases were between 11-40 years, in above the age of 61 years- 4 cases were noticed. It correlates the study of Akhyani $\mathrm{M}$ et al. 2,5,3,4,6,7

The most common cause of these dermatitis in Spanish, Middle Eastern and Indian studies includes psoriasis and eczema:2,5,4 The trigger for precipitating erythroderma in psoriatic patients are withdrawal of systemic or topical glucocorticoids, use of systemic medications like lithium and antimalarials, phototherapy, burns, infection and systemic illnesses. ${ }^{1} \mathrm{~A}$ major challenge lies in determining the aetiology of erythroderma as pre-existing dermatosis is the single most common cause of erythroderma as determined by most published series.8,3,4,9,10 Eight of our patients were diagnosed as pityriasis rubra pilaris which is a common cause of erythroderma.5,11,12,9 Our series has a high percentage of erythroderma secondary to pre-existing dermatoses that are mentioned as the most common cause of adult erythroderma in majority of the studies. $5,7,12,9$ 
In our study, pre-existing dermatoses constitute thirtyfour cases. Psoriasis was commonly seen in sixteen cases, airborne allergic contact dermatitis was noticed in nine cases, two cases each was seen in scabies and lichen planus, one case each was noticed in ichthyosis, phototoxic dermatitis, pityriasis rubra pilaris, pemphigus foliaceus, dermatophytosis respectively, this correlates with study of Pal and Haroon et.al

Like many other series, $11,8,7$ diffuse scaling and pruritus was found in almost all our patients. Although we examined each patient in several occasions, we found a low percentage of lymphadenopathy, visceral enlargement, oedema and mucosal involvement in comparison with Pal and Haroon's series. $^{4}$

In our study, common symptoms like fever, malaise were noticed in seventeen cases. Other symptoms noticed were moderate itching, generalised erythema, scaling, burning sensation. Six patients had oedema of limbs, lymphadenopathy was noticed in fourteen cases.

Many drugs can cause erythroderma. Among the more commonly implicated are pyrazolone derivatives, carbamazepine, hydantoin derivatives, cimetidine, lithium salts and gold salts.2,7,12 Carbamazepine was the most common drug causing erythroderma.12,9,10 Drug reactions were the most common cause of erythroderma in HIV patients. ${ }^{6}$

In the present study, the drug causing erythroderma was seen in five cases. Carbamazepine-induced reaction was seen in two cases. One each in Dapsone, Analgin, cotrimoxazole induced respectively. Dapsone induced erythroderma patient had jaundice, hepatomegaly and oral mucosa involvement correlates with study.

Mild anaemia, raised ESR, leucocytosis and hypoalbuminaemia were common laboratory findings; however, nothing contributory to underlying cause of erythroderma could be found. ${ }^{1}$

In the present study, anaemia was observed in fourteen cases. Normocytic normochromic anaemia was noticed in six cases, megaloblastic anaemia was seen in five cases, dimorphic anaemia was seen three in cases. Leucocytosis More than 11000 cells/cu. mm seen in seventeen cases, neutrophils and eosinophils counts were high. ESR more than $15 \mathrm{~mm} / \mathrm{hr}$. was seen in nineteen cases.

Erythrodermic psoriasis (17.2\%) was seen in patients with pre-existing psoriasis who gave a history of irregular use of medications or stoppage of all treatment. In $10.3 \%$ cases, no cause of erythroderma could be established. Skin biopsy was not rewarding in these cases and showed only mild spongiosis and acanthosis. Histopathology showed specific change in $8.4 \%$ patients. ${ }^{1}$

In our study, histopathology showed specific change in six cases $(14 \%)$ - three in psoriasis patients, two in lichen planus patients, one in pemphigus foliaceus which correlates with the study of Nadia Shirazi et al.

\section{Idiopathic Erythroderma}

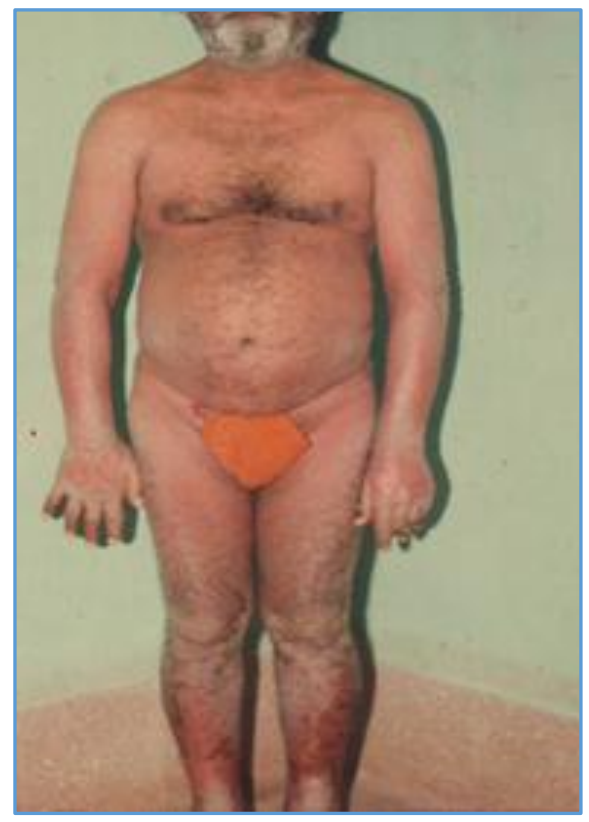

\section{Erythroderma due to Airborne allergic contact Dermatitis}

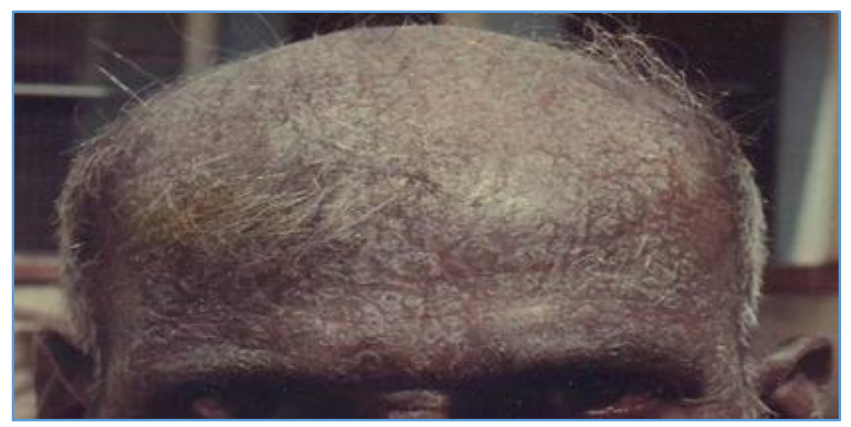

\section{Erythroderma due to Dapsone}

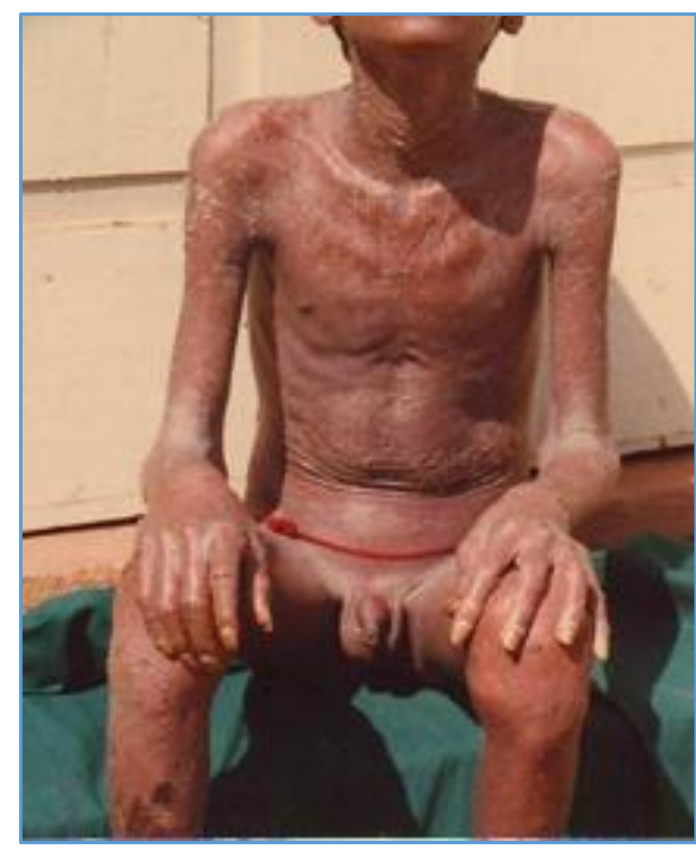




\section{Erythroderma due to Psoriasis}

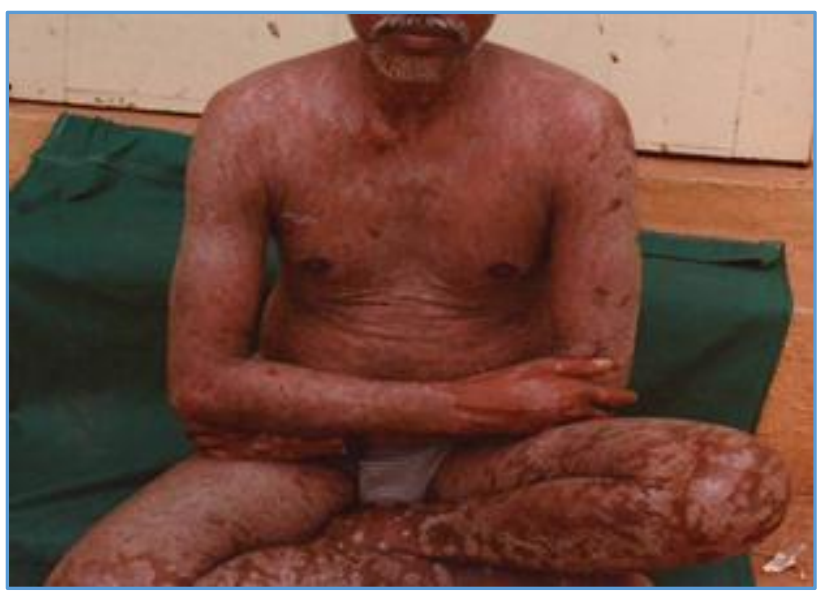

Erythroderma is occasionally associated with internal malignancies, even patients with previous history of known dermatoses whose clinicopathological features are inconclusive should be investigated carefully to rule out malignant neoplastic causes. ${ }^{2}$

\begin{tabular}{|l|c|c|c|c|}
\hline \multicolumn{1}{|c|}{ Cause } & $\begin{array}{c}\text { Pal, Haroon } \\
\text { et al }\end{array}$ & Sehgal et al & Akhyani M et al & Present Study \\
\hline 1) Pre-existing dermatoses & $74.4 \%$ & $52.5 \%$ & $57.9 \%$ & $79.07 \%$ \\
\hline 2) Drug reaction & $5.5 \%$ & $24.7 \%$ & $21.6 \%$ & $11.62 \%$ \\
\hline 3) Malignancy & $5.5 \%$ & ---- & $11.3 \%$ & $2.34 \%$ \\
\hline 4) Idiopathic & $14.6 \%$ & $22.5 \%$ & $7.2 \%$ & $6.97 \%$ \\
\hline \multicolumn{2}{r}{} \\
\hline
\end{tabular}

\begin{tabular}{|c|c|c|c|c|}
\hline \multirow[b]{2}{*}{ Sl. No. } & \multirow[b]{2}{*}{ Aetiology } & \multirow[b]{2}{*}{$\begin{array}{l}\text { No. of } \\
\text { Cases }\end{array}$} & \multicolumn{2}{|c|}{ Histopathology } \\
\hline & & & Specific & $\begin{array}{c}\text { Non- } \\
\text { specific }\end{array}$ \\
\hline 1 & Psoriasis & 16 & 3 & 13 \\
\hline 2 & $\begin{array}{c}\text { Airborne allergic contact } \\
\text { dermatitis, Phototoxic dermatitis }\end{array}$ & 10 & --- & 10 \\
\hline 3 & Pemphigus foliaceus & 1 & 1 & --- \\
\hline 4 & PRP, Scabies. Ichthyosis & 4 & --- & 4 \\
\hline 5 & Lichen planus & 2 & 2 & --- \\
\hline 6 & Dermatophytosis & 1 & --- & 1 \\
\hline 7 & Drug induced & 5 & --- & 5 \\
\hline 8 & Idiopathic, Malignancy & 4 & --- & 4 \\
\hline \multicolumn{5}{|c|}{ Table 2. Histopathology of Skin Biopsy of Erythroderma Cases } \\
\hline
\end{tabular}

\section{REFERENCES}

[1] Shiraz N, Jindal R, Jain A, et al. Erythroderma: a clinicoetological study of 58 cases in tertiary hospital of north India. Asian Journal of Medical Science 2015;6(6):20-4.

[2] Akhyani M, Ghodsi ZS, Toosi S, et al. Erythroderma a clinical study of 97 cases. BMC Dermatol 2005;5:1-5.

[3] Richard B, William D, Tinothy G. Diseases of the skin. 9th edn. New York, WB Saunders 2000:252-3.

[4] Pal S, Haroon TS. Erythroderma: a clino-etiological study of 90 cases. Int J Dermatol 1998;37(2):104-7.

[5] Botella-Estrada R, Sanmartin O, Oliver V, et al. Erythroderma. A clinicopathological study of 56 cases. Arch Dermatol 1994;130(12):1503-7.

[6] Morar N, Dlova N, Gupta AK, et al. Erythroderma: a comparison between HIV positive and negative patients. Int J Dermatol 1999;38(12):895-900.
In our study, erythroderma due to malignancy was noted in one case Hodgkin's lymphoma, idiopathic erythroderma was seen in three cases, this correlates with study of Akhyani M et. al.

\section{CONCLUSION}

Erythroderma can be caused by a variety of underlying dermatoses, infections, and systemic diseases. A high percentage of erythroderma cases secondary to pre-existing dermatoses had a low percentage of idiopathic cases. Since clinical features are identical irrespective of the underlying cause and laboratory findings, pointing to a specific diagnosis may not be rewarding. The clinician should take detailed history supported by ancillary investigations. However, for definite clinicopathological correlation, multiple skin biopsies may be needed followed by close followup and repeated evaluation. Despite the homogeneity of clinical expression of erythroderma, diagnostic histopathological features of the underlying disease are retained in most cases. Hospitalisation is needed for initial evaluation and treatment. Prognosis of erythroderma is dependent on underlying aetiology. 